All figures are precursor ion spectra:

- Figure $\mathrm{S} 1: \mathrm{m} / \mathrm{z}=119$ from protonated $\mathrm{Phe}, \mathrm{E}_{\mathrm{lab}}=15 \mathrm{~V}$

- Figure $\mathrm{S} 2: \mathrm{m} / \mathrm{z}=143$ from protonated $\mathrm{Trp}, \mathrm{E}_{\mathrm{lab}}=15 \mathrm{~V}$

- Figure S3: $\mathrm{m} / \mathrm{z}=117$ from protonated Trp, $\mathrm{E}_{\text {lab }}=20 \mathrm{~V}$

- Figure S4: $\mathrm{m} / \mathrm{z}=115$ from protoanted Trp, $\mathrm{E}_{\text {lab }}=20 \mathrm{~V}$

- Figure S5: $\mathrm{m} / \mathrm{z}=82$ from protonated His, $\mathrm{E}_{\mathrm{lab}}=15 \mathrm{~V}$

- Figure S6 m/z=68 from protonated His, $\mathrm{E}_{\mathrm{lab}}=15 \mathrm{~V}$

- Figure S7: $\mathrm{m} / \mathrm{z}=66$ from protonated His, $\mathrm{E}_{\mathrm{lab}}=15 \mathrm{~V}$

- Cartesian coordinates $(\AA)$ and electronic energies (hartree) for structures and transition states shown in Figures 5 - 7 of the main paper. 


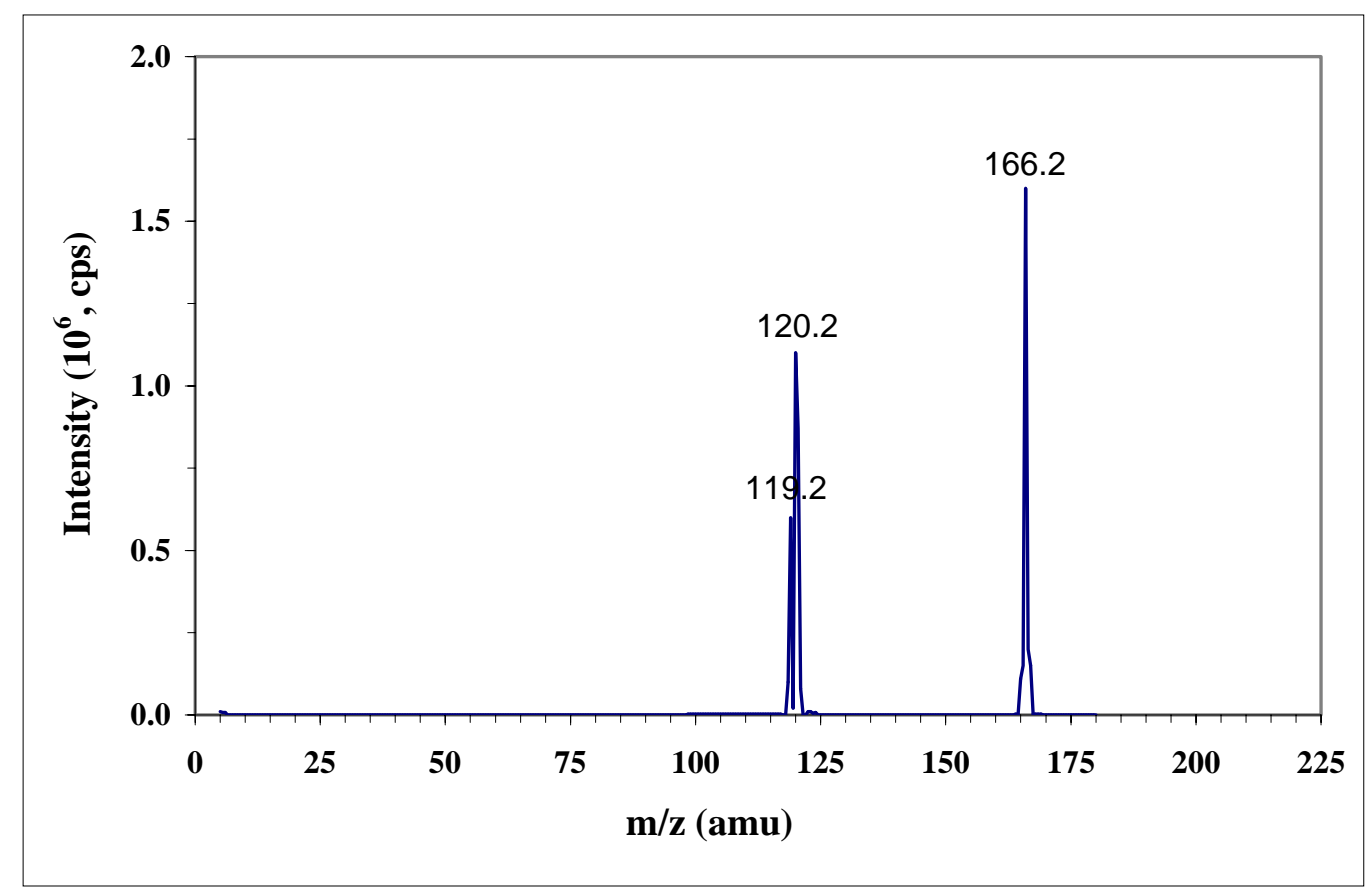

Figure S1

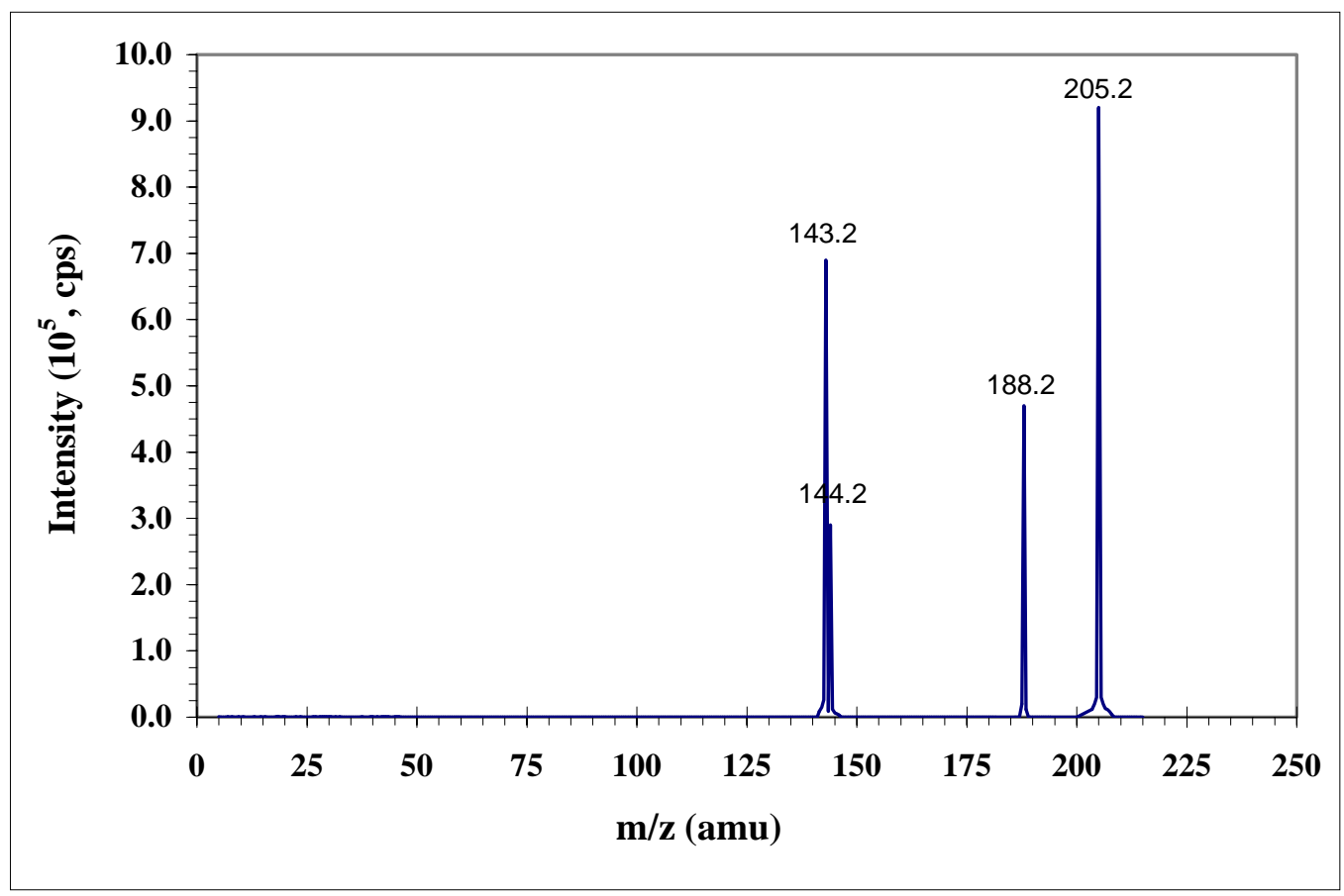

Figure S2 


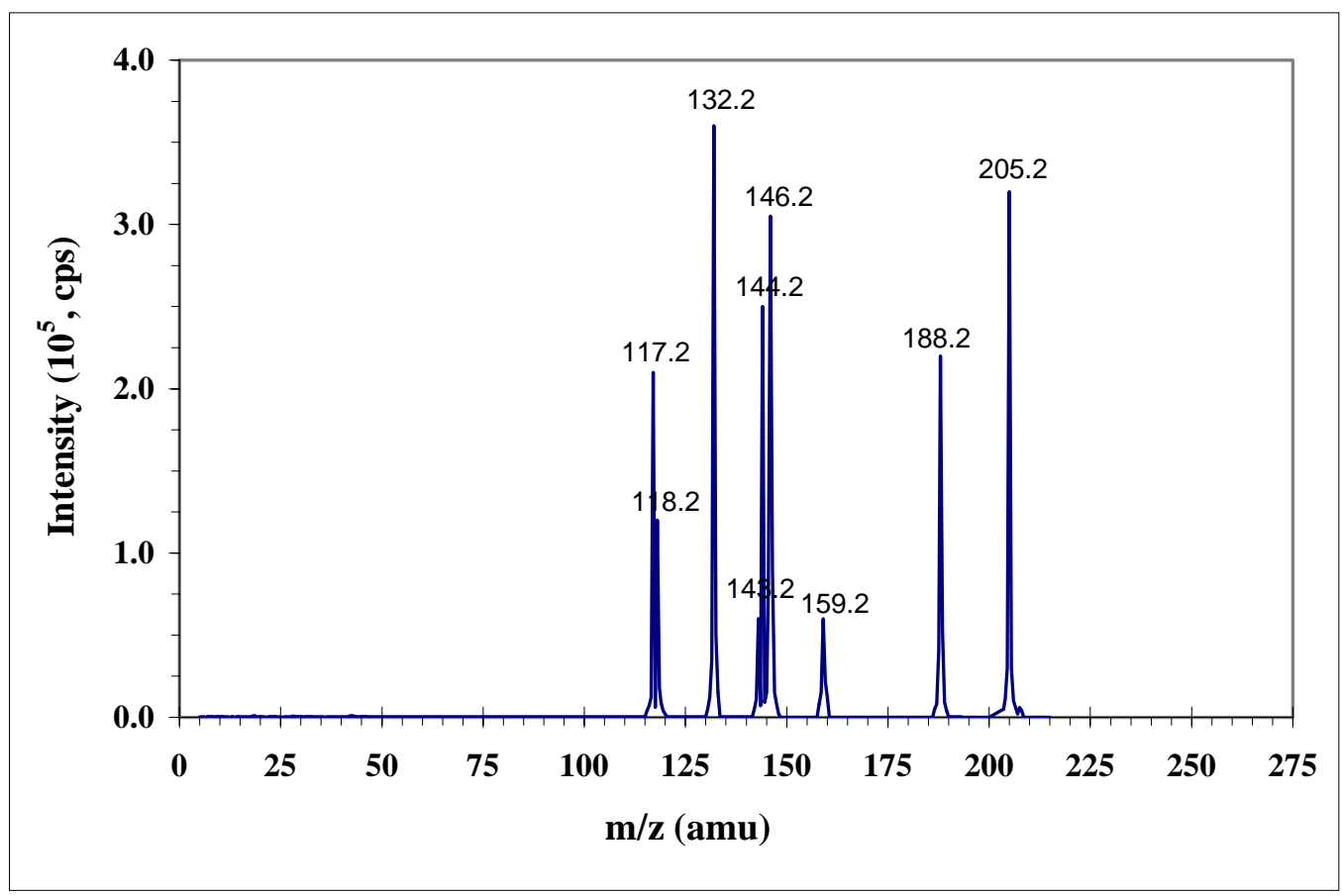

Figure S3

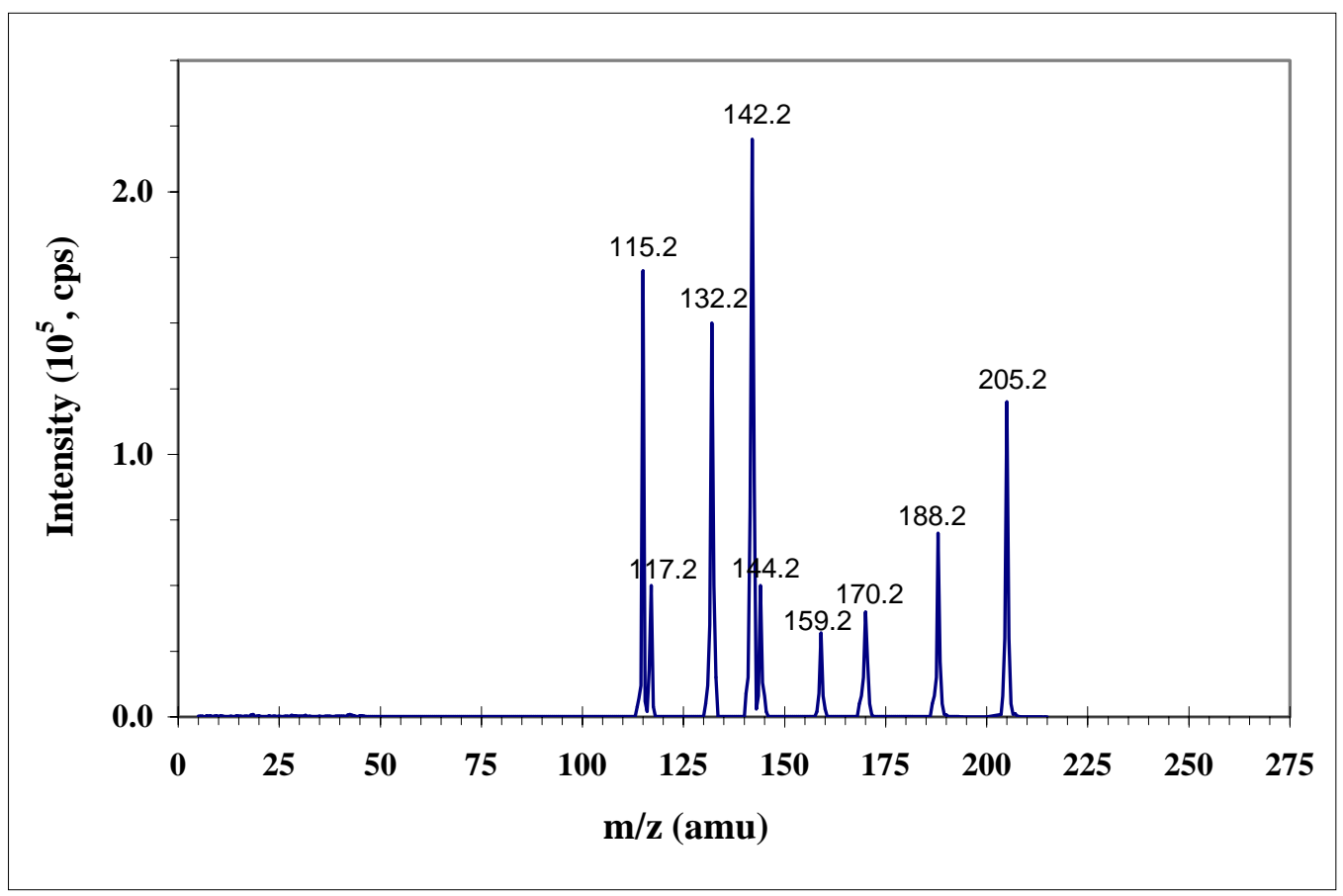

Figure S4 


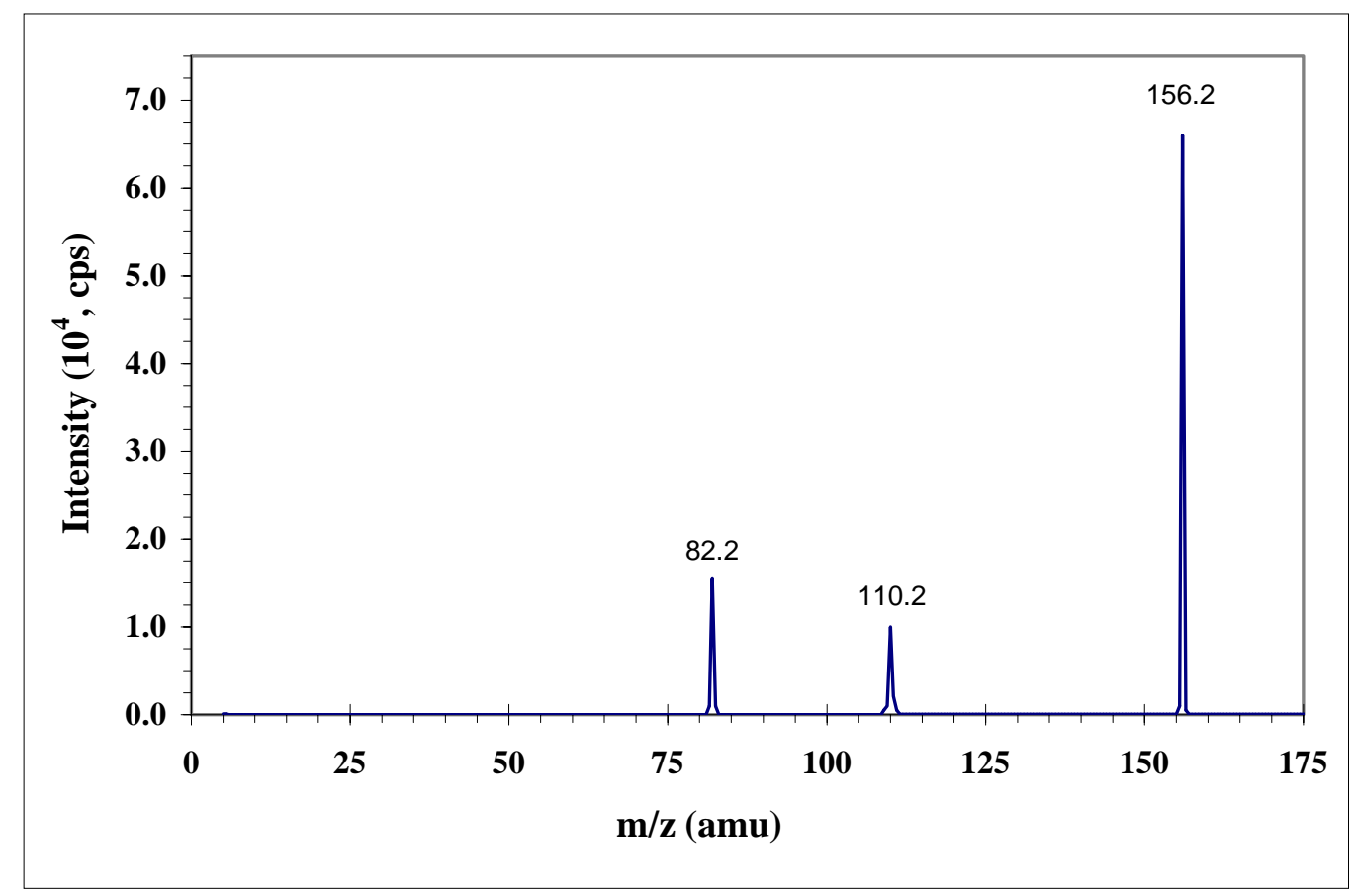

Figure S5

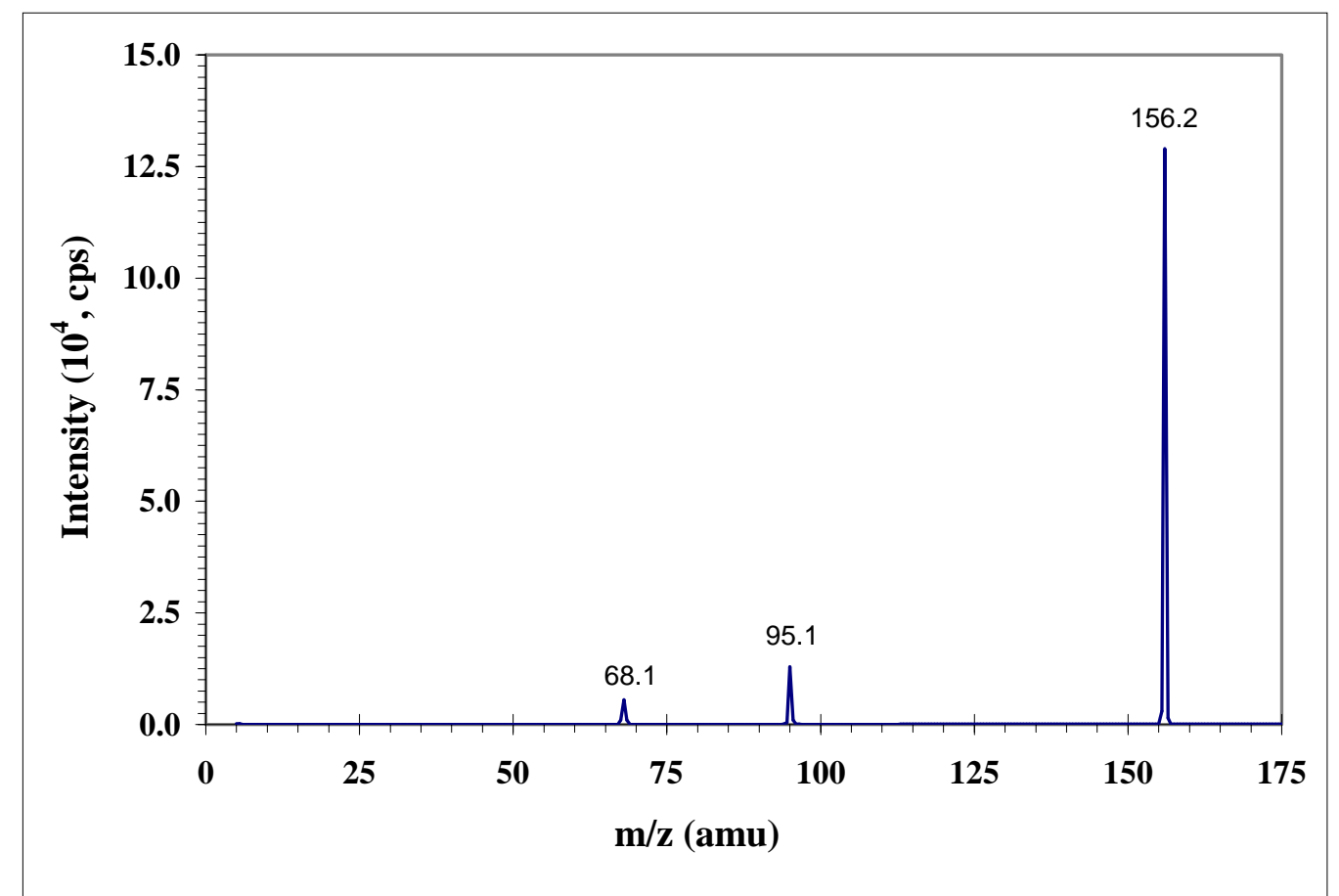

Figure S6 


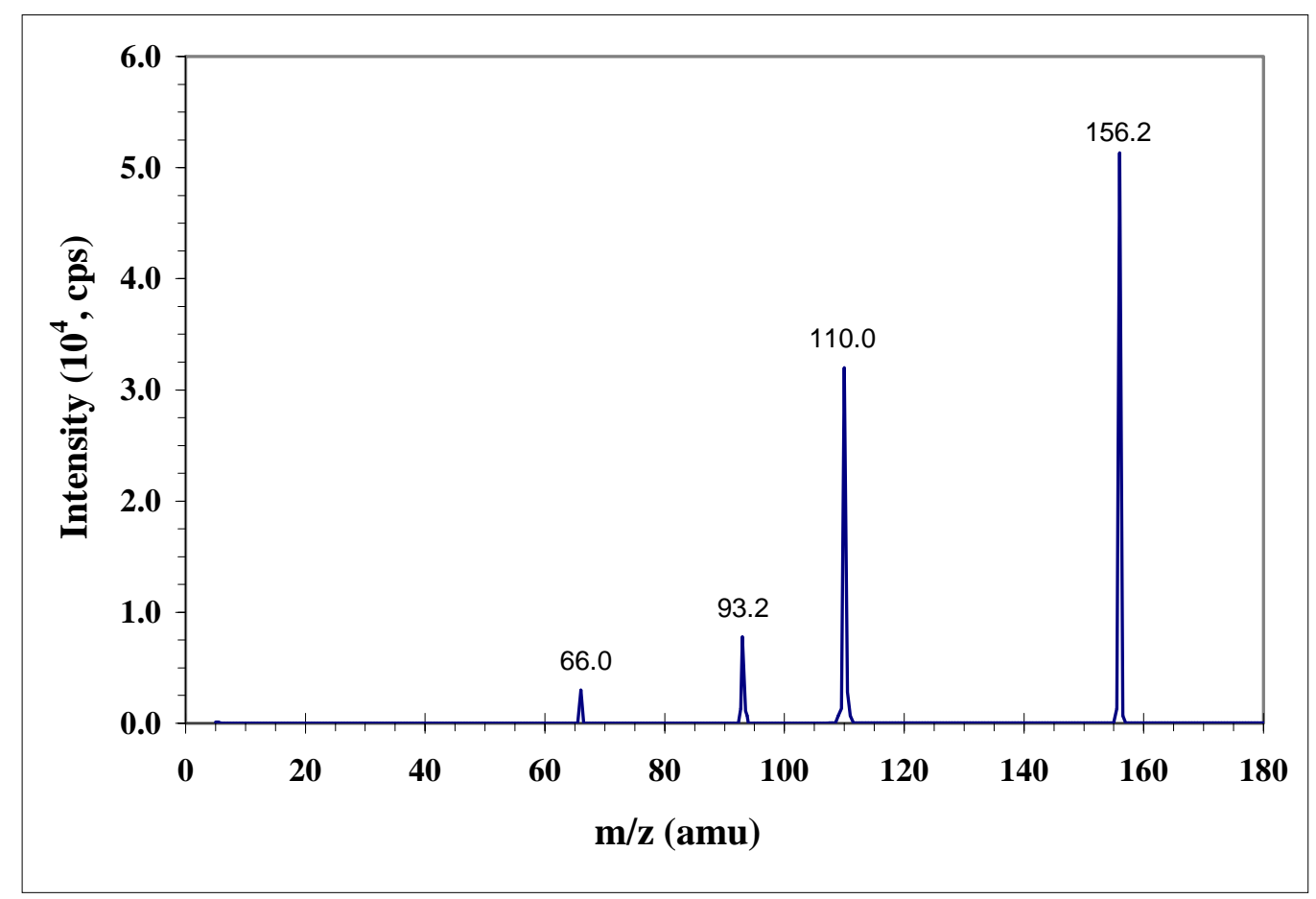

Figure S7 
Figure 5 (all energies in hartree and coordinates in $\AA$ ) Structure $\mathrm{I}$. $\mathrm{E}_{\mathrm{t}}=-555.1809335$

$\begin{array}{rrrr}6 & -3.034516 & -1.090899 & -0.432591 \\ 6 & -1.691721 & -0.922433 & -0.793247 \\ 6 & -1.009500 & 0.264758 & -0.473686 \\ 6 & -1.696306 & 1.281157 & 0.210515 \\ 6 & -3.035333 & 1.112661 & 0.567192 \\ 6 & -3.704968 & -0.075192 & 0.251059 \\ 1 & -3.554848 & -2.005374 & -0.702557 \\ 1 & -1.193194 & -1.697785 & -1.374481 \\ 1 & -1.192098 & 2.217042 & 0.444271 \\ 1 & -3.560150 & 1.912201 & 1.082199 \\ 6 & 0.457435 & 0.418293 & -0.822139 \\ 1 & 0.685549 & 1.429673 & -1.173591 \\ 6 & 1.370314 & 0.162653 & 0.407723 \\ 1 & 1.212733 & 0.935481 & 1.163386 \\ 1 & -4.748686 & -0.199780 & 0.524213 \\ 6 & 2.848188 & 0.060096 & 0.029463 \\ 8 & 3.428382 & -1.005830 & -0.005601 \\ 8 & 3.357807 & 1.247545 & -0.275584 \\ 1 & 4.292497 & 1.149328 & -0.550687 \\ 7 & 1.022618 & -1.171283 & 1.037694 \\ 1 & 0.025508 & -1.390963 & 0.866200 \\ 1 & 1.634190 & -1.893703 & 0.618827 \\ 1 & 1.192076 & -1.185098 & 2.049290 \\ 1 & 0.737033 & -0.272325 & -1.627187\end{array}$

Structure II. $E_{\mathrm{t}}=-555.166096$

$\begin{array}{lrrr}6 & 2.793683 & -0.758813 & -1.006288 \\ 6 & 1.517351 & -1.166768 & -0.616728 \\ 6 & 0.857923 & -0.525933 & 0.446890 \\ 6 & 1.508509 & 0.522290 & 1.119605 \\ 6 & 2.788499 & 0.929616 & 0.727089 \\ 6 & 3.430261 & 0.291873 & -0.336286 \\ 1 & 3.294841 & -1.266127 & -1.825454 \\ 1 & 1.045621 & -2.001853 & -1.132404 \\ 1 & 1.024518 & 1.012467 & 1.961909 \\ 1 & 3.281521 & 1.737422 & 1.259754 \\ 6 & -0.530747 & -0.975355 & 0.875208 \\ 1 & -0.792796 & -0.520677 & 1.839018 \\ 6 & -1.615294 & -0.626136 & -0.167002 \\ 1 & -1.342512 & -1.034433 & -1.145514 \\ 1 & 4.425034 & 0.605444 & -0.638603 \\ 6 & -1.950522 & 0.879513 & -0.356026 \\ 8 & -3.114744 & 1.228375 & -0.341252 \\ 8 & -0.956805 & 1.719217 & -0.567887 \\ 1 & -0.071122 & 1.307954 & -0.455942 \\ 7 & -2.957161 & -1.245161 & 0.201259 \\ 1 & -3.021071 & -1.457670 & 1.203717 \\ 1 & -3.664397 & -0.502085 & -0.016144 \\ 1 & -3.168085 & -2.101950 & -0.31980 \odot \\ 1 & -0.537009 & -2.064827 & 1.014995 \\ & & & \\ \text { TS (II } \rightarrow \text { III }) . & \mathrm{E}_{\mathrm{t}}=-555.130501 \\ 6 & -2.899868 & 0.837685 & -\odot .800944 \\ 6 & -1.665482 & 1.362725 & -\odot .381420 \\ 6 & -0.855267 & 0.639212 & 0.481392\end{array}$




$\begin{array}{rrrr}6 & -1.286313 & -0.683913 & 0.897767 \\ 6 & -2.580918 & -1.164783 & 0.484695 \\ 6 & -3.357582 & -0.425739 & -0.386711 \\ 1 & -3.517593 & 1.428438 & -1.473089 \\ 1 & -1.354544 & 2.341154 & -0.738169 \\ 1 & -0.939296 & -1.032947 & 1.877258 \\ 1 & -2.922343 & -2.128278 & 0.853661 \\ 6 & 0.536875 & 1.097474 & 0.842257 \\ 1 & 0.814513 & 0.727695 & 1.836743 \\ 6 & 1.637854 & 0.672472 & -0.184519 \\ 1 & 1.315861 & 0.989606 & -1.184581 \\ 1 & -4.318471 & -0.795830 & -0.729370 \\ 6 & 1.843104 & -0.860680 & -0.306315 \\ 8 & 3.087336 & -1.239903 & -0.455036 \\ 8 & 0.924632 & -1.684721 & -0.318077 \\ 1 & -0.446596 & -1.269369 & 0.239516 \\ 7 & 2.946358 & 1.275195 & 0.071451 \\ 1 & 3.131924 & 1.429722 & 1.061184 \\ 1 & 3.638616 & -0.410645 & -0.396710 \\ 1 & 3.087143 & 2.152586 & -0.421761 \\ 1 & 0.556124 & 2.191790 & 0.882853\end{array}$

Structure III. $6 \quad 2.581445$

$6 \quad 1.318934$

$6 \quad 0.729830$

$6 \quad 1.514755$

$6 \quad 2.830869$

$6 \quad 3.336995$

$1 \quad 3.007544$

$1 \quad 0.795039$

$1 \quad 1.670085$

$1 \quad 3.388721$

$6-0.621865$

$1-0.841167$

$6 \quad-1.783722$

$1 \quad-1.615978$

14.304088

$6 \quad-1.709174$

$8 \quad-2.869959$

$8 \quad-0.636969$

$1 \quad 0.896834$

$7 \quad-3.122834$

$1 \quad-3.274629$

$1 \quad-3.575666$

$1-3.416383$

$1-0.611621$

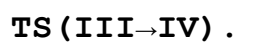

$6 \quad 2.662339$

$6 \quad 1.345215$

$6 \quad 0.938525$

$6 \quad 2.029802$

$6 \quad 3.419375$

$6 \quad 3.715875$

$1 \quad 2.923671$

$1 \quad 0.608058$
$E_{t}=-555.1327551$

$-0.495191 \quad-1.227664$

$-0.982668 \quad-0.887237$

$-0.627228 \quad 0.322946$

$0.184178 \quad 1.289833$

$0.691130 \quad 0.841638$

$0.361656-0.380956$

$-0.783448 \quad-2.186167$

$-1.627582-1.586996$

$-0.421874 \quad 2.206019$

$1.321826 \quad 1.529079$

$-1.147069 \quad 0.723987$

$-0.870899 \quad 1.761837$

$-0.642352-0.190148$

$-1.003929-1.212450$

$0.727333-0.710200$

$0.898801-0.284161$

$1.518415-0.407856$

$1.475576-0.265396$

$1.022092 \quad 1.651502$

$-1.048557 \quad 0.194912$

$-1.069517 \quad 1.201640$

$0.827338-0.352208$

$-1.937463-0.199664$

$-2.243451 \quad 0.669812$

$\mathrm{t}==-555.089594$
$-1.160889$
$-0.781052$
$\odot .879473$
0.306817
$\odot .801724$
1.103073
$-0.028213$
0.558687
$-0.706513$
$-0.510138$
$-0.628688$
$-2.003996$
142385
$-1.349909$
1. 359745 


\begin{tabular}{|c|c|c|c|}
\hline & 2.043300 & 2.101019 & -0.230234 \\
\hline & 4.189021 & 1.080717 & -1.191031 \\
\hline & $-\odot .395108$ & $\odot .665082$ & -0.281190 \\
\hline & $-\odot .897519$ & $-\odot .308322$ & -1.296932 \\
\hline & -1.598334 & 0.236956 & 0.56347 \\
\hline & -1.302509 & -0.536923 & 1.28324 \\
\hline & 4.729772 & $-\odot .889245$ & $\odot .219452$ \\
\hline & -2.504492 & -0.487300 & -0.441266 \\
\hline & -3.758304 & $-\odot .66190 \odot$ & -0.197650 \\
\hline & -1.951878 & -0.874571 & -1.496061 \\
\hline & 1.761054 & 1.315411 & -1.75077 \\
\hline & -2.421837 & 1.196226 & 1.28599 \\
\hline & -2.519100 & 2.084715 & $\odot .79608$ \\
\hline & -3.962210 & -0.158168 & $\odot .632936$ \\
\hline & -2.072834 & 1.389094 & 2.22102 \\
\hline & $-\odot .545569$ & 1.606369 & -0.81750 \\
\hline
\end{tabular}

TS (III $\rightarrow$ IV) ' .

$6 \quad 2.812608$

$6 \quad 1.459605$

$6 \quad 0.958122$

$6 \quad 1.946482$

$6 \quad 3.394398$

$6 \quad 3.799851$

$1 \quad 3.153389$

$1 \quad 0.779863$

$1 \quad 1.750789$

14.110567

$6-0.388086$

$1-0.684742$

$6 \quad-1.559486$

$1-1.281706$

14.852303

$6 \quad-2.770974$

$8 \quad-2.609320$

$8 \quad-3.730413$

$1 \quad 1.730391$

$7 \quad-1.847682$

$1 \quad-1.871119$

$1-3.394554$

$1 \quad-2.714682$

$1-0.693981$

Structure IV.

$6 \quad-2.941368$

$6 \quad-1.446317$

$6 \quad-0.947874$

$6 \quad-1.927828$

$6-3.256483$

$6-3.781535$

$1-3.312863$

$1-1.039655$

$1-1.574259$

$1 \quad-3.957240$

$6 \quad 0.364176$

$1 \quad 0.651101$

$6 \quad 1.485199$
$E_{t}=-555.0803646$

$0.774198 \quad 1.105507$

$0.684759 \quad 0.863578$

$-0.116131-0.197056$

$-0.970285-0.950789$

$-0.739083-0.673792$

$0.084711 \quad 0.320187$

$1.404881 \quad 1.923251$

$1.252700 \quad 1.490108$

$-0.934715 \quad-2.031697$

$-1.289071-1.278603$

$-0.138024-0.620686$

$-0.978618 \quad-1.252751$

$0.558324 \quad 0.094222$

$1.042302 \quad 1.031230$

$0.230678 \quad 0.540752$

$-0.339688 \quad 0.311575$

$-1.125202 \quad 1.382499$

$-0.341644-0.426869$

$-2.022073-0.682137$

$1.580396-0.971653$

$2.552917-0.659110$

$-1.700683 \quad 1.479897$

$1.356657-1.474559$

$0.964336-1.446326$

\begin{tabular}{rr}
\multicolumn{2}{c}{$E_{t}=-555.1298558$} \\
0.896154 & 0.930018 \\
0.753222 & 0.823242 \\
-0.283761 & -0.174464 \\
-0.878186 & -1.061812 \\
-0.626800 & -0.930930 \\
0.255884 & 0.098058 \\
1.572107 & 1.695677 \\
0.559651 & 1.826721 \\
-1.560014 & -1.830945 \\
-1.107714 & -1.608272 \\
-0.649873 & -0.289809 \\
-1.330885 & -1.090301 \\
-0.115920 & 0.532997
\end{tabular}




$\begin{array}{rrrr}1 & 1.161881 & 0.617063 & 1.273550 \\ 1 & -4.855949 & 0.391170 & 0.176457 \\ 6 & 2.619583 & 0.450598 & -0.331672 \\ 8 & 3.604879 & -0.204912 & -0.606617 \\ 8 & 2.355180 & 1.685106 & -0.743618 \\ 1 & 3.069122 & 1.995190 & -1.337247 \\ 7 & 2.169756 & -1.271777 & 1.311126 \\ 1 & -1.041237 & 1.738179 & 0.537271 \\ 1 & 2.863982 & -1.697924 & 0.673207 \\ 1 & 2.684441 & -0.950713 & 2.140478 \\ 1 & 1.476594 & -1.973130 & 1.593629\end{array}$

Structure v. $\quad E_{t}=-670.8722197$

\begin{tabular}{|c|c|c|c|}
\hline 6 & -3.379397 & -0.754684 & 0.699121 \\
\hline & -2.059861 & $-\odot .898719$ & 1.144076 \\
\hline & -1.025532 & -0.238866 & $\odot .496018$ \\
\hline & -1.338193 & 0.591408 & $-\odot .691189$ \\
\hline & -2.754141 & ๑.718557 & -1.083750 \\
\hline & -3.740404 & ๑.059438 & $-0.4 c$ \\
\hline & -4.165432 & -1.287588 & 1.23000 \\
\hline & -1.852737 & -1.524738 & 2.00662 \\
\hline & $-\odot .799150$ & 0.180122 & -1.56726 \\
\hline & -2.984682 & 1.332514 & -1.95080 \\
\hline & 0.366323 & $-\odot .232767$ & 1.04169 \\
\hline & $\odot .453674$ & -0.960971 & 1.854816 \\
\hline & 1.511165 & -0.428488 & 0.01407 \\
\hline & 1.263939 & 0.123997 & -0.90026 \\
\hline 1 & -4.781778 & $\odot .140151$ & $-\odot .69955$ \\
\hline 0 & 1.535006 & -1.903850 & $-\odot .40918$ \\
\hline 0 & 2.769646 & -2.422208 & -0.477 \\
\hline 8 & $\odot .519050$ & -2.512605 & -0.681052 \\
\hline & -0.836857 & 1.577742 & $-\odot .58462$ \\
\hline 7 & 2.738708 & ๑.175919 & 0.52130 \\
\hline 1 & 3.507738 & ๑.033578 & -0.13178 \\
\hline 1 & 2.705894 & -3.347295 & -0.7890 \\
\hline 1 & 3.030766 & -0.243386 & 1.40384 \\
\hline 1 & ๑.512919 & 0.768836 & 1.47316 \\
\hline 8 & 1.123676 & 2.595938 & -0.02594 \\
\hline 1 & 1.922193 & 2.101842 & ๑. 25648 \\
\hline 6 & 1.410684 & 4.000994 & -0.06205 \\
\hline 1 & 2.208447 & 4.224871 & $-\odot .7805$ \\
\hline 1 & 0.496613 & 4.506389 & -0.3819 \\
\hline & 694609 & 4.375371 & \\
\hline
\end{tabular}

TS $(\mathrm{V} \rightarrow \mathrm{VI}) . \mathrm{E}_{\mathrm{t}}=-670.8483868$

$\begin{array}{rrrr}6 & -3.268480 & 0.331044 & 1.069752 \\ 6 & -1.914990 & 0.420801 & 1.266939 \\ 6 & -0.987766 & 0.008774 & 0.260041 \\ 6 & -1.550053 & -0.631544 & -0.983552 \\ 6 & -3.036692 & -0.617678 & -1.135121 \\ 6 & -3.847865 & -0.171002 & -0.151863 \\ 1 & -3.936598 & 0.654017 & 1.864495 \\ 1 & -1.529736 & 0.818486 & 2.202353 \\ 1 & -1.215792 & -1.685582 & -0.984476 \\ 1 & -3.441325 & -1.016724 & -2.061452 \\ 6 & 0.389454 & 0.226258 & 0.436290\end{array}$




$\begin{array}{rrrr}1 & 0.700535 & 0.405066 & 1.470753 \\ 6 & 1.465267 & -0.460617 & -0.418804 \\ 1 & 1.123715 & -0.533446 & -1.454863 \\ 1 & -4.927789 & -0.190410 & -0.258331 \\ 6 & 1.640975 & -1.903596 & 0.068921 \\ 8 & 2.661423 & -2.031398 & 0.941096 \\ 8 & 0.908422 & -2.808214 & -0.263416 \\ 1 & -1.082018 & -0.211849 & -1.886871 \\ 7 & 2.706154 & 0.355627 & -0.427822 \\ 1 & 3.343448 & 0.026621 & -1.153380 \\ 1 & 2.698607 & -2.959096 & 1.248013 \\ 1 & 3.206959 & 0.246856 & 0.455764 \\ 1 & 0.574391 & 1.602591 & -0.087633 \\ 8 & 1.197665 & 2.452907 & -0.621268 \\ 1 & 2.066042 & 1.887908 & -0.643646 \\ 6 & 1.325313 & 3.714497 & 0.098338 \\ 1 & 2.010790 & 4.347411 & -0.466806 \\ 1 & 0.334166 & 4.166675 & 0.122713 \\ 1 & 1.700470 & 3.544112 & 1.111174\end{array}$

Structure VI. $E_{t}=-670.8828371$

$\begin{array}{rrrr}6 & -3.467055 & 0.063759 & 1.108354 \\ 6 & -2.163547 & -0.182977 & 1.393515 \\ 6 & -1.168064 & -0.344251 & 0.346618 \\ 6 & -1.669296 & -0.398897 & -1.091215 \\ 6 & -3.114308 & -0.025555 & -1.292538 \\ 6 & -3.951157 & 0.176701 & -0.259761 \\ 1 & -4.175953 & 0.193249 & 1.921918 \\ 1 & -1.830880 & -0.241247 & 2.427023 \\ 1 & -1.535978 & -1.426320 & -1.469164 \\ 1 & -3.464124 & 0.036296 & -2.319806 \\ 6 & 0.144747 & -0.470183 & 0.688350 \\ 1 & 0.412096 & -0.512175 & 1.744250 \\ 6 & 1.279134 & -0.665524 & -0.268782 \\ 1 & 0.965606 & -0.569749 & -1.310001 \\ 1 & -4.996689 & 0.416969 & -0.428301 \\ 6 & 1.978814 & -2.011796 & -0.047822 \\ 8 & 2.999717 & -2.115074 & 0.599602 \\ 8 & 1.307661 & -3.012981 & -0.617705 \\ 1 & -1.045427 & 0.226098 & -1.747105 \\ 7 & 2.353459 & 0.389461 & -0.026521 \\ 1 & 3.082048 & 0.361864 & -0.748601 \\ 1 & 1.750642 & -3.860234 & -0.409795 \\ 1 & 2.826479 & 0.179475 & 0.861969 \\ 1 & 0.395211 & 3.035149 & 0.417050 \\ 8 & 1.270168 & 2.921986 & 0.012879 \\ 1 & 1.936153 & 1.357165 & 0.009103 \\ 6 & 1.874882 & 4.215667 & -0.219894 \\ 1 & 2.843236 & 4.027413 & -0.686244 \\ 1 & 1.255874 & 4.807983 & -0.900085 \\ 1 & 2.022219 & 4.748724 & 0.724276\end{array}$

Figure 6

Structure $\mathrm{XI} . \mathrm{E}_{\mathrm{t}}=-498.5692752$

$6-3.030141 \quad-1.039383 \quad-0.268372$ 


$\begin{array}{rrrr}6 & -1.710786 & -1.306872 & 0.039489 \\ 6 & -0.784164 & -0.232235 & 0.270607 \\ 6 & -1.253986 & 1.121914 & 0.175834 \\ 6 & -2.574905 & 1.37270 \odot & -0.132593 \\ 6 & -3.459635 & 0.297109 & -0.353846 \\ 1 & -3.731290 & -1.849019 & -0.443211 \\ 1 & -1.357812 & -2.332186 & 0.110597 \\ 1 & -0.574061 & 1.949536 & 0.345882 \\ 1 & -2.936723 & 2.393282 & -0.205842 \\ 6 & 0.530967 & -0.564218 & 0.585069 \\ 1 & 0.777311 & -1.625364 & 0.611161 \\ 6 & 1.654539 & 0.344910 & 0.869255 \\ 1 & 1.397507 & 1.402296 & 0.899788 \\ 1 & 2.083885 & 0.057284 & 1.844172 \\ 1 & -4.498227 & 0.507204 & -0.596199 \\ 6 & 2.819843 & 0.168755 & -0.139673 \\ 8 & 3.408481 & 1.098299 & -0.617781 \\ 8 & 3.072584 & -1.131952 & -0.366302 \\ 1 & 3.840491 & -1.209881 & -0.968310\end{array}$

$\operatorname{TS}(X I \rightarrow X I I) \quad E_{t}=-498.5296346$

$\begin{array}{rrrr}6 & -2.968142 & -0.906883 & 0.027298 \\ 6 & -1.620232 & -1.263786 & 0.096485 \\ 6 & -0.626038 & -0.272989 & 0.077014 \\ 6 & -0.993650 & 1.080657 & -0.023499 \\ 6 & -2.339042 & 1.432275 & -0.098759 \\ 6 & -3.326639 & 0.439315 & -0.069580 \\ 1 & -3.732691 & -1.677475 & 0.047897 \\ 1 & -1.344244 & -2.313369 & 0.172871 \\ 1 & -0.238628 & 1.863006 & -0.063131 \\ 1 & -2.620318 & 2.477923 & -0.179784 \\ 6 & 0.799085 & -0.692755 & 0.156214 \\ 1 & 0.896424 & -1.774211 & 0.271018 \\ 6 & 1.777234 & 0.042257 & 1.100738 \\ 1 & 1.442631 & 1.043179 & 1.402157 \\ 1 & 2.100803 & -0.502285 & 1.994776 \\ 1 & -4.374850 & 0.718659 & -0.123211 \\ 6 & 2.891664 & 0.182466 & 0.128001 \\ 8 & 3.966027 & 0.402965 & -0.196824 \\ 8 & 1.614024 & -0.294258 & -1.064768 \\ 1 & 1.665021 & -0.948425 & -1.793326\end{array}$

Structure $\mathrm{XII} \quad \mathrm{E}_{\mathrm{t}}=-498.5360101$

$\begin{array}{llrr}6 & -2.843791 & 1.096745 & -0.012058 \\ 6 & -1.479729 & 1.273414 & 0.228249 \\ 6 & -0.611882 & 0.171501 & 0.192209 \\ 6 & -1.118575 & -1.109315 & -0.090523 \\ 6 & -2.482360 & -1.281350 & -0.324011 \\ 6 & -3.344307 & -0.178478 & -0.288363 \\ 1 & -3.513418 & 1.950737 & 0.025245 \\ 1 & -1.095885 & 2.265164 & 0.457953 \\ 1 & -0.456747 & -1.972986 & -0.109414 \\ 1 & -2.874009 & -2.272705 & -0.531688 \\ 6 & 0.852986 & 0.375381 & 0.461108 \\ 1 & 1.035274 & 1.389056 & 0.838387 \\ 6 & 1.690772 & 0.225034 & -0.887778 \\ 1 & 1.403450 & -0.700510 & -1.406986\end{array}$




$\begin{array}{rrrr}1 & 1.567439 & 1.077967 & -1.568202 \\ 1 & -4.405870 & -0.315823 & -0.472397 \\ 6 & 3.079553 & 0.099599 & -0.555047 \\ 8 & 4.162237 & 0.015691 & -0.248151 \\ 8 & 1.466730 & -0.590962 & 1.300278 \\ 1 & 0.852028 & -0.853922 & 2.007371\end{array}$

TS (XII $\rightarrow X I I I+X I V) \quad E_{t}=-498.5249381$

\begin{tabular}{|c|c|c|c|}
\hline & 2. 619801 & -1.263525 & ๑. 338029 \\
\hline 6 & 1.289764 & -1.076140 & ๑. 699248 \\
\hline & ๑.627030 & 0.118999 & ๑.348061 \\
\hline & 1.309091 & 1.120917 & -0.373885 \\
\hline & 2.638541 & ๑.922952 & -0.731298 \\
\hline & 3.292411 & $-\odot .266319$ & -0.379985 \\
\hline & 3.135377 & -2.176574 & 0.619135 \\
\hline 1 & $\odot .769534$ & -1.844550 & 1.267577 \\
\hline 1 & ๑.803956 & 2.047132 & $-\odot .629343$ \\
\hline 1 & 3.171519 & 1.695179 & -1.277502 \\
\hline 6 & $-\odot .751280$ & ๑. 301115 & 0.772993 \\
\hline$\perp$ & -1.125282 & -0.356731 & 1.556367 \\
\hline 6 & -1.761425 & -0.690607 & -0.780873 \\
\hline$\perp$ & -1.397137 & -0.111237 & -1.630048 \\
\hline 1 & -1.400282 & -1.718370 & -0.736351 \\
\hline 1 & 4.331172 & -0.413319 & -0.661512 \\
\hline 6 & -3.101073 & -0.559518 & -0.563960 \\
\hline 8 & -4.180970 & -0.353581 & -0.231748 \\
\hline 8 & -1.225738 & 1.549388 & 0.757162 \\
\hline & -2.012354 & 1.664776 & 1.31839 \\
\hline
\end{tabular}

Structure XIII. $E_{t}=-345.918278$

\begin{tabular}{|c|c|c|c|}
\hline & 1.719719 & -1.182638 & $\odot .000000$ \\
\hline & 1.376051 & 0.158471 & \\
\hline & $\odot . ๑ \odot \odot \odot \odot \odot$ & $\odot .533719$ & $\odot$. \\
\hline & -1.019375 & -0.465062 & ๑. \\
\hline & -0.656833 & -1.798213 & $\odot . \odot \odot \odot$ \\
\hline & 0.706404 & -2.154770 & $\odot .0000$ \\
\hline & 2.762746 & -1.481934 & \\
\hline & 2.146807 & 0.924928 & \\
\hline & -2.063922 & -0.170729 & $\odot .0000$ \\
\hline & -1.418174 & -2.571638 & $\odot .000 \odot$ \\
\hline & $-\odot .300935$ & 1.901243 & $\odot .0000$ \\
\hline & 0.496581 & 2.646187 & $\odot .0000$ \\
\hline & ๑.979355 & -3.206674 & $\odot . \odot \odot$ \\
\hline & -1.530300 & 2.325582 & \\
\hline & -1.611179 & 3.298713 & $\odot . \odot \odot \odot$ \\
\hline
\end{tabular}

\begin{tabular}{lccr}
\multicolumn{2}{c}{ Structure XIV } & \multicolumn{2}{c}{$\mathrm{E}_{\mathrm{t}}=-152.6072473$} \\
6 & $0.000 \odot \odot \odot$ & $0.00 \odot \odot \odot \odot$ & 0.101816 \\
8 & $0.000 \odot \odot \odot$ & $0.000 \odot \odot \odot$ & 1.273288 \\
6 & $0.00 \odot \odot \odot \odot$ & $0.00 \odot \odot \odot \odot$ & -1.215067 \\
1 & $0.00 \odot \odot \odot \odot$ & 0.940405 & -1.753403 \\
1 & $0.000 \odot \odot \odot$ & -0.940405 & -1.753403
\end{tabular}

Figure 7 (all energies in hartree and coordinates in $\AA$ )

Structure $\mathrm{xV} \quad \mathrm{E}_{\mathrm{t}}=-302.7231115$

$\begin{array}{llll}6 & -1.622527 & 0.003482 & -0.000077\end{array}$ 


$\begin{array}{rrrr}6 & -0.275454 & -0.017501 & -0.00 \odot 455 \\ 7 & 0.506350 & -1.188723 & -0.000448 \\ 6 & 0.587072 & 1.125302 & -0.00 \odot 376 \\ 6 & 1.729008 & -0.783362 & 0.00 \odot 365 \\ 7 & 1.816012 & 0.631701 & 0.00 \odot 244 \\ 1 & 0.360375 & 2.183982 & -0.00 \odot 423 \\ 1 & 2.618462 & -1.400824 & 0.00 \odot 996 \\ 1 & 2.676645 & 1.175311 & 0.00 \odot 702 \\ 6 & -2.911236 & -0.002155 & 0.000361 \\ 1 & -3.476178 & -0.006467 & 0.934843 \\ 1 & -3.477013 & -0.007441 & -0.933605\end{array}$

\begin{tabular}{|c|c|c|c|}
\hline \multicolumn{2}{|c|}{$T S(X V \rightarrow X V I)$} & \multicolumn{2}{|c|}{$E_{t}==-302.6173711$} \\
\hline 6 & -1.606715 & $-\odot .046397$ & 0.000000 \\
\hline & $-\odot .283607$ & $-\odot .044039$ & -0.000001 \\
\hline & 0.516381 & -1.183305 & -0.000001 \\
\hline & $\odot .596423$ & 1.189678 & 0.000001 \\
\hline & 1.700241 & -1.269562 & $\odot .000 \odot \odot 1$ \\
\hline & 1.836507 & 1.165546 & $\odot .000000$ \\
\hline & $\odot .045359$ & 2.135825 & $\odot .000003$ \\
\hline & 2.778106 & 0.815996 & -0.000009 \\
\hline & -2.903180 & -0.000674 & 0.000000 \\
\hline & -3.467881 & 0.015147 & $\odot .932722$ \\
\hline & -3.467881 & $\odot .015149$ & -0.932721 \\
\hline & 2.623113 & -1.831837 & $\odot .000003$ \\
\hline
\end{tabular}

\section{Structure XVI}

$$
-1.465177
$$

$-0.136630$

0.535046

$\odot .693998$

1.160334

1.953965

0.073499

1.725926

2. 482444

$-2.761318$

$-3.326032$

$-3.326146$
$E_{t}=-302.6417529$

$-\odot .068020 \quad 0.000226$

$-0.099640 \quad-0.000090$

$1.117755 \quad 0.000074$

$-1.355136-0.000575$

$2.081918-0.000517$

$-1.475420 \quad 0.000400$

$-2.253531-0.001884$

$3.001589 \quad 0.001191$

$-0.596415 \quad 0.001737$

$-0.119989 \quad 0.000185$

$-0.140725-0.932819$

$-0.142066 \quad 0.933088$

TS (XVI $\rightarrow X V I I+X V I I I) \quad E_{t}=-302.5916497$

$-1.594183$

$6-0.301969-0.244201-0.000037$

$7 \quad-0.593499 \quad-1.212822 \quad-0.000018$

$\begin{array}{llll}6 & 0.580905 & 1.383152 & -0.000014\end{array}$

$\begin{array}{llll}6 & 1.838265 & -1.213411 & 0.000016\end{array}$

$\begin{array}{llll}7 & 1.777598 & 1.422048 & 0.000023\end{array}$

$\begin{array}{llll}1 & -0.215260 & 2.121725 & -0.000054\end{array}$

$\begin{array}{llll}1 & 2.467056 & -2.102400 & -0.000001\end{array}$

$\begin{array}{llll}1 & 2.315062 & -0.163455 & 0.000049\end{array}$

$\begin{array}{llll}6 & -2.897077 & -0.020376 & 0.000019\end{array}$

$\begin{array}{llll}1 & -3.460080 & 0.017715 & 0.933129\end{array}$

$\begin{array}{llll}1 & -3.460094 & 0.017803 & -0.933079\end{array}$

Structure XVII $E_{t}=-209.2063746$

$\begin{array}{llll}6 & -1.273435 & -0.000230 & -0.000435\end{array}$

$\begin{array}{llll}6 & -0.000837 & -0.000149 & 0.000704\end{array}$ 


$\begin{array}{rrrr}6 & -2.587309 & -0.000 \odot 55 & -\odot .00 \odot \odot 47 \\ 1 & -3.151610 & -\odot .137389 & -0.923506 \\ 1 & -3.150904 & 0.139159 & 0.923575 \\ 7 & 1.219390 & -0.000099 & 0.000115 \\ 6 & 2.482228 & 0.000148 & -0.000349 \\ 1 & 3.021678 & -0.938126 & 0.136022 \\ 1 & 3.021225 & 0.938767 & -0.136133\end{array}$

$\begin{array}{lccr}\text { Structure XVIII } & \mathrm{E}_{\mathrm{t}}=-93.4286169 \\ 6 & 0.00 \odot \odot \odot \odot & 0.00 \odot \odot \odot \odot & -0.502169 \\ 7 & 0.00 \odot \odot \odot \odot & 0.00 \odot \odot \odot \odot & 0.655306 \\ 1 & 0.0 \odot \odot \odot \odot \odot & 0.0 \odot \odot \odot \odot \odot & -1.574126\end{array}$

TS $(X V \rightarrow X I X) \quad E_{t}=-302.6563658$

$\begin{array}{rrrr}6 & 1.560310 & 0.010510 & 0.000022 \\ 6 & 0.236587 & -0.047471 & -0.000 \odot \odot 3 \\ 7 & -0.538566 & 1.111668 & 0.00 \odot 119 \\ 6 & -0.616025 & -1.275744 & -0.000154 \\ 6 & -1.550824 & 1.665592 & 0.000169 \\ 7 & -1.87740 \odot & -1.151533 & -0.00 \odot 156 \\ 1 & -0.05180 \odot & -2.212930 & -0.00 \odot 253 \\ 1 & -2.432650 & 2.286540 & 0.00 \odot 227 \\ 1 & -2.374082 & -2.044841 & -0.00 \odot 264 \\ 6 & 2.857464 & 0.015436 & 0.000 \odot 41 \\ 1 & 3.42260 \odot & 0.020066 & 0.932656 \\ 1 & 3.422626 & 0.020279 & -0.932557\end{array}$

\begin{tabular}{lrrr}
\multicolumn{2}{c}{ Structure XIX } & \multicolumn{2}{c}{$E_{\mathrm{t}}=-302.6568585$} \\
6 & -1.511153 & -0.045196 & -0.000044 \\
6 & -0.185474 & -0.097275 & -0.000075 \\
7 & 0.543204 & 1.089558 & -0.000163 \\
6 & 0.664608 & -1.323903 & 0.000093 \\
6 & 1.326918 & 1.927392 & -0.000198 \\
7 & 1.926763 & -1.198635 & 0.000328 \\
1 & 0.095029 & -2.258621 & $0.0000 \odot 1$ \\
1 & 2.035077 & 2.741137 & -0.000240 \\
1 & 2.410009 & -2.099868 & 0.000452 \\
6 & -2.808549 & -0.048072 & -0.000008 \\
1 & -3.374025 & -0.048475 & -0.932499 \\
1 & -3.373954 & -0.048308 & 0.932529
\end{tabular}

Structure $\mathrm{xx} \quad \mathrm{E}_{\mathrm{t}}=-302.6034744$

$\begin{array}{rrrr}6 & 1.343763 & -0.875883 & 0.063813 \\ 6 & 0.846569 & 0.254006 & 0.216895 \\ 7 & -1.861381 & -0.529708 & 0.035925 \\ 6 & 0.276756 & 1.548402 & 0.393401 \\ 6 & -2.955761 & -0.866977 & -0.123068 \\ 7 & 0.629447 & 2.442683 & -0.461108 \\ 1 & -0.379731 & 1.694424 & 1.254187 \\ 1 & -3.973328 & -1.180318 & -0.270119 \\ 1 & 0.223729 & 3.358447 & -0.22830 \odot \\ 6 & 1.902071 & -2.076571 & -0.116417 \\ 1 & 2.329227 & -2.355341 & -1.080584 \\ 1 & 1.943256 & -2.805893 & 0.693358\end{array}$

Structure XXI $\quad E_{\mathrm{t}}==-209.1584746$

$\begin{array}{llll}6 & -1.212212 & 0.057862 & -0.000054\end{array}$ 


$\begin{array}{rrrr}6 & 0.014533 & 0.247102 & -0.000347 \\ 6 & 1.422048 & 0.462312 & -0.0000 \odot 5 \\ 7 & 2.156048 & -0.597516 & 0.000 \odot 79 \\ 1 & 1.782997 & 1.494654 & 0.000151 \\ 1 & 3.157531 & -0.359127 & 0.000548 \\ 6 & -2.534313 & -0.170287 & 0.000105 \\ 1 & -3.248531 & 0.654570 & 0.000878 \\ 1 & -2.924682 & -1.189414 & -0.000321\end{array}$

